\title{
The role of human endogenous retroviral long terminal repeat sequences in human cancer (Review)
}

\author{
HONG-LIAN YU ${ }^{1}$, ZHAN-KUI ZHAO ${ }^{2}$ and FAN ZHU ${ }^{1}$ \\ ${ }^{1}$ Department of Medical Microbiology, School of Medicine; ${ }^{2}$ Department of Urology, \\ Zhongnan Hospital, Wuhan University, Wuhan, Hubei 430071, P.R. China
}

Received March 17, 2013; Accepted May 30, 2013

DOI: $10.3892 /$ ijmm.2013.1460

\begin{abstract}
Human endogenous retrovirus (HERV) and solitary long terminal repeats (LTRs) constitute $8 \%$ of the human genome. Although most HERV genes are partially deleted and not intact, HERV LTRs comprise features including promoters, enhancers, selective splicer sites and polyadenylation sites in order to regulate the expression of neighboring genes. Owing to the genetic instability of LTRs, their wide distributions along human chromosomes are not only non-random, but are also correlated with gene density. Considerable evidence indicates that HERV LTRs regulate the expression of their adjacent viral and cellular genes in placental development and tumorigenesis. However, the regulatory mechanism of HERV LTRs on the expression of its neighboring cancer-associated genes in human cancers remains to be elucidated. Insertional mutagenesis, recombination and polymorphism are three principal factors of LTR that contribute to its genetic instability. Moreover, genetic instability, hypomethylation, transactivation and the antisense transcript of LTRs enhance the activity of LTRs and regulate the expression of their adjacent genes in human cancers. Therefore, in the present review, we examined the mechanism of HERV LTRs in tumorigenesis in combination with the structure and function of LTRs.
\end{abstract}

\section{Contents}

1. Introduction

2. Functions of HERV LTR in physiology

3. Activation of HERV LTR in tumorigenesis

4. Effect mechanisms of HERV LTR in cancer

5. Conclusion

Correspondence to: Professor Fan Zhu, Department of Medical Microbiology, School of Medicine, Wuhan University, 185 Donghu Road, Wuhan, Hubei 430071, P.R. China

E-mail: zhufan@hotmail.com

Key words: genetic instability, methylation, long terminal repeat, human endogenous retrovirus, tumorigenesis

\section{Introduction}

Almost half of the human genome is derived from transposable elements (Fig. 1A) (1). The majority of transposable elements consist of retrotransposon. Retrotransposon is divided according to whether or not it contains long terminal repeats (LTRs). Human endogenous retroviruses (HERV) and solitary LTR represent $\sim 8 \%$ of the human genome as compared to non-LTR retrotransposon elements which represent $34 \%$ of the human genome. HERVs are classified into Class I (HERV-W and HERV-H), Class II (HERV-K) and Class III (HERV-L and HERV-S) following phylogenetic analyses of the POL gene with respect to the established classification of animal retroviruses $(\gamma-, \beta$ - and spuma retrovirus) (2). Standard HERVs share several general structures with retroviruses, such as 5'LTR, GAG, POL, ENV and 3'LTR (Fig. 1B).

The number and location of LTRs from several main HERV families are shown in Table I. Different HERV families possess distinct copies. The HERV-K family is considered the most active HERV family and has numerous solitary LTRs $(3,4)$. Although HERV LTRs are widely distributed in the human genome, their distributions along the human chromosome are not only non-random but also associated with gene density $(5,6)$. HERV LTRs are enriched in the region of transcription units. LTRs can be located in the sense and antisense orientation of its adjacent gene (Fig. 2). HERV LTRs within introns of annotated genes exhibit a strong orientation bias, such that HERV LTRs are usually oriented opposite to the transcription direction of the corresponding host genes (7). LTRs are uniformly distributed among sequences of a variety of GC content as they are less abundant only in the most GC-rich regions (6). In addition, LTRs have apparently been inserted randomly into these regions. LTR may be located at any region of the adjacent genes, including 5'UTR, intron, exon and 3'UTR (Fig. 2). These distributions provide favorable conditions for LTRs regulating the expression of their neighboring genes in different ways.

Although the majority of HERV genes are highly defective with large deletions, stop codons and frameshifts in the open reading fames, HERV LTR still retain their functions, such as promoter, enhancer and transcriptional factor-binding site and potentially regulate their neighboring viral and cellular genes. Non-LTR retrotransposon has been reported to cause human cancer through insertional mutagenesis of 
Table I. Distribution of LTRs from HERV families.

\begin{tabular}{lcccc}
\hline $\begin{array}{l}\text { Family } \\
\text { name }\end{array}$ & $\begin{array}{c}\text { Complete } \\
\text { provirus }\end{array}$ & $\begin{array}{c}\text { Solitary LTR } \\
\text { (copy) }\end{array}$ & $\begin{array}{c}\text { Intergration time } \\
\text { (million years ago) }\end{array}$ & Refs. \\
\hline HERV-I & 85 & $101-4$ & $30-48$ & $(71)$ \\
HERV-H & 100 & 1000 & $30-35$ & $(72,73)$ \\
HERV-W & 115 & 1100 & 30 & $(74)$ \\
HERV-L & 575 & 6000 & 70 & $(75)$ \\
ERV-9 & 70 & $3000-4000$ & $33-40$ & $(76)$ \\
HERV-K & $20-50$ & $10000-25000$ & $8-15$ & $(2,3,77)$ \\
\hline
\end{tabular}

HERV, human endogenous retrovirus; LTR, long terminal repeats.

A

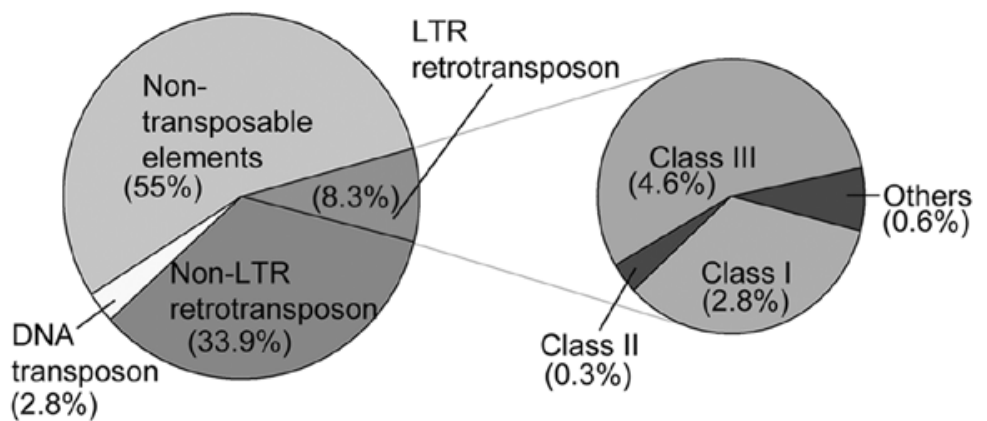

B

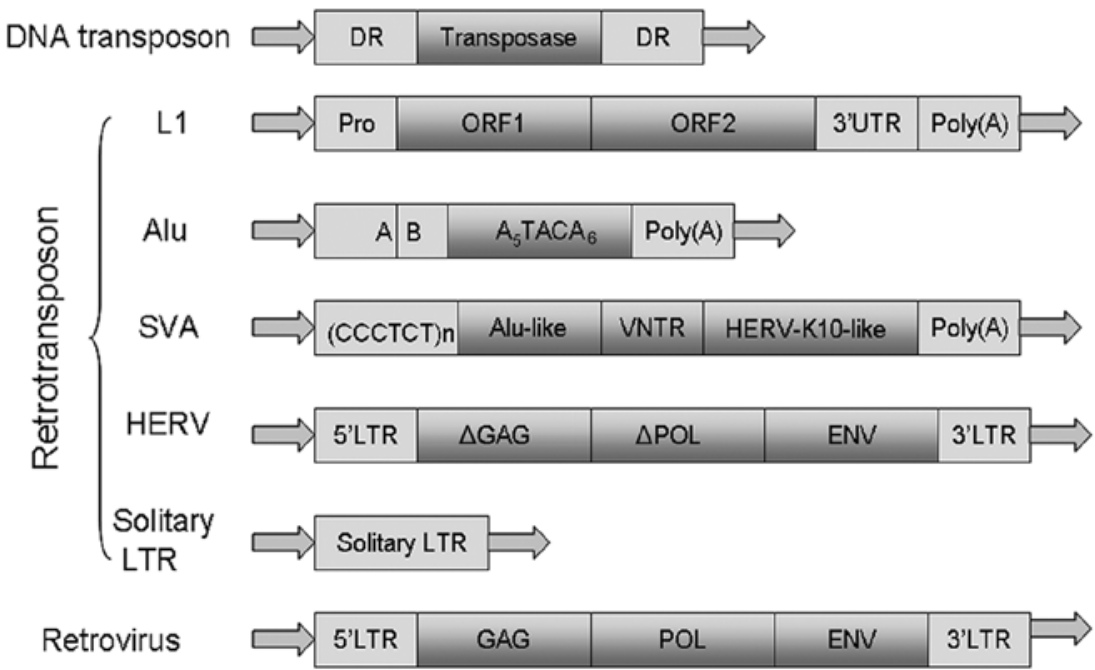

Figure 1. The classification and structure of transposable elements in the human genome. (A) Approximately $45 \%$ of the human genome is considered to be composed of transposable elements. Transposable elements can be classified as: DNA transposon, non-long terminal repeats (LTR) retrotransposons and LTR retrotransposon. LTR retrotransposon consists of three types of HERV (Class I, II and III) according to the classification of retroviruses. (B) Structure of the various transposons. The DNA transposon comprises the transposase gene and two short direct repeats (DRs). The retrotransposon includes the canonical L1 element, Alu element, SVA element, HERV and solitary LTR. The L1 element consists of two open reading frames (ORF) flanked by 5' and 3'UTRs and ends with a polyadenylation [poly(A)] tail. The Alu element consists of two related monomers (A and B grey boxes), A-rich linker region $\left(\mathrm{A}_{5} \mathrm{TACA}_{6}\right)$ and ends with a poly(A). The SVA element has a composite structure consisting of a (CCCTCT)n hexamer repeat region, an Alu-like region, a variable number of tandem repeats (VNTR) region, HERV-K10-like sequence and a poly(A) tail. HERV contains 5'LTR-GAG-POL-ENV-3'LTR. The majority of these genes are not intact. Solitary LTR is formed though excision provirus by homologous recombination. The retrovirus contains 5 ' and 3 'LTR flanking sequences that produce proteins (GAG, POL and ENV) necessary for mobilization.

genes, retrotransposition-associated mutagenesis, non-allelic homologous recombination and hypomethylation of retroelement promoters (8). Previous studies have reported that LTR elements affected adjacent genes to contribute to cancer (9-11).
However, the molecular mechanism of LTR in tumorigenesis has yet to be reported. Herein, we briefly review the characteristics of HERV LTRs, their distribution, as well as the available evidence for LTR biological significance and function in 


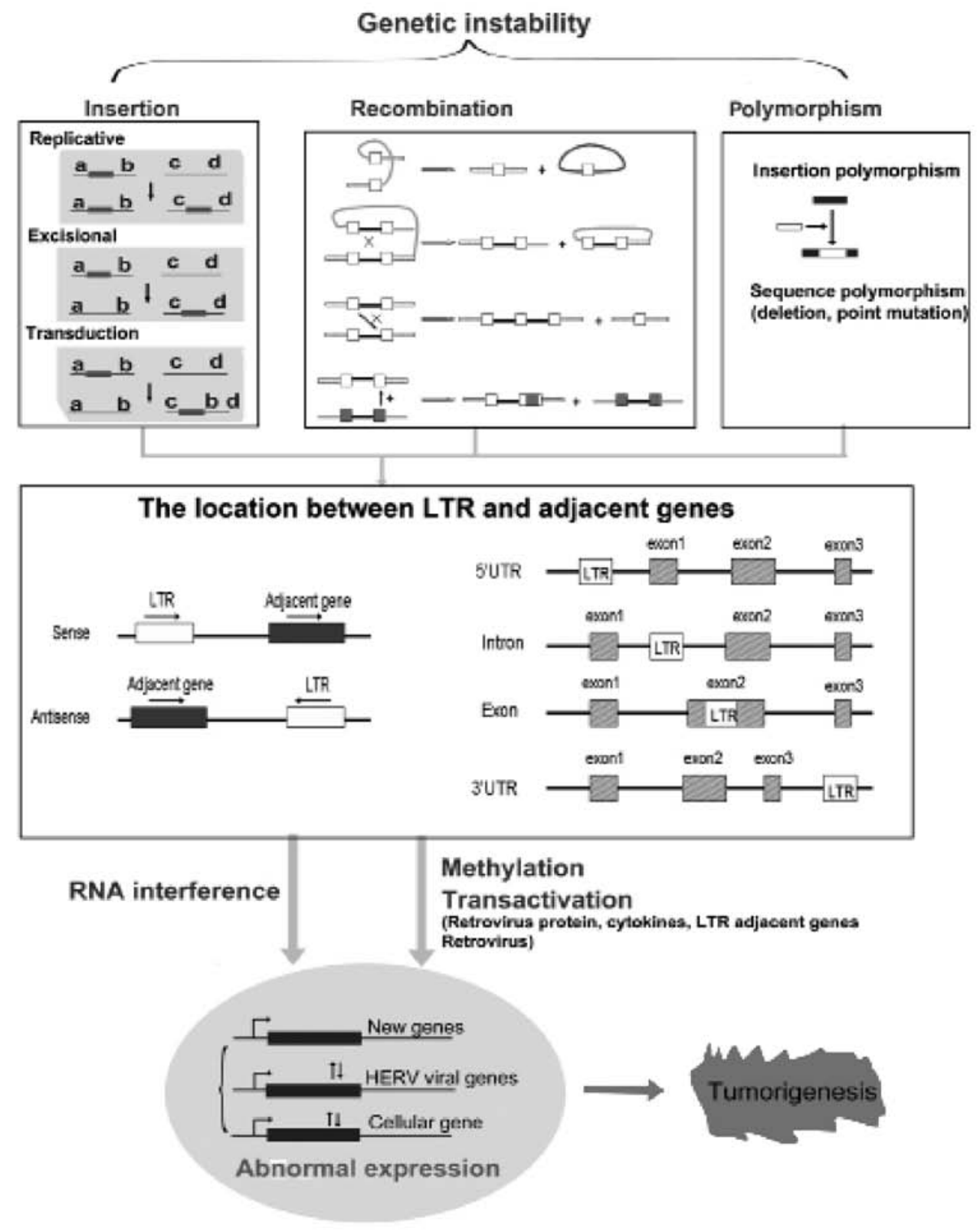

Figure 2. The role of human endogenous retrovirus (HERV) long terminal repeats (LTR) in human tumorigenesis. Abnormal expression of new, HERV viral and cellular genes are associated with human tumorigenesis in four ways including genetic instability (insertion, recombination and polymorphism), methylation, transactivation and RNA interference. Insertion is divided into: replicative, excisional and transduction. Recombination between the two LTRs of a single provirus, homologous recombination between two HERV on the same chromosome, recombination between the 3' and 5'LTRs of one HERV, and gene conversion between non-homologous genes is evident. Genetic instability of HERV LTR causes the diverse distributions of LTR. LTR can be located in the sense and antisense orientation of its adjacent gene. LTR can also be located at any region of its adjacent gene, such as 5'LTR, exon, intron and 3'LTR. These wide distributions of LTR can regulate the expression of their adjacent genes to become involved in tumorigenesis by methylation, transactivation and RNA interference.

physiological and pathological processes. The focus of this review is on the role of HERV LTR in human tumorigenesis.

\section{Functions of HERV LTR in physiology}

HERV LTR elements are retained in the vicinity of genes in order to regulate their expression during primate evolution. LTR elements and nearby genes appear to have forged a mutually beneficial relationship. LTR elements can benefit from genes by surviving from one generation to the next, while genes can benefit from the characteristics of LTR. For example, genes could also obtain their enhancer (12), promoter (sometimes bidirectional) (12-16), polyadenylation [poly(A)] sites $(17,18)$ from LTR. Alteration of the expression of adjacent genes usually results from LTR. For example, an explanation of LTR promoter activity involves the expression of amylase in the human parotid glands, where integration of HERV-E in reverse orientation upstream of the pancreatic amylase gene promotes its expression and release into saliva (19). In addition, bidirectional promoter activity is observed in HERV-K LTR for the neighboring reporter gene (20). As an alternative promoter LTR is capable of enhancing the transcription from a native promoter of apolipoprotein CI and the endothelin B receptor genes (13). HERV-K LTR revealed its tissue-specific 
Table II. Examples of tumor-associated genes regulated by HERV LTRs.

\begin{tabular}{lllc}
\hline $\begin{array}{l}\text { LTR adjacent viral } \\
\text { and cellular genes }\end{array}$ & \multicolumn{1}{c}{ Associated cancer } & \multicolumn{1}{c}{ LTR } & Refs. \\
\hline Rec & Melanoma and germ cell tumor & HERV-K & $(36,37)$ \\
Np9 & Various tumor tissues & HERV-K & $(34)$ \\
Env & Testicular cancer & HERV-W & $(78)$ \\
& Breast cancer & HERV-K & $(51)$ \\
Reverse transcriptase & Breast cancer & HERV-K & $(79)$ \\
Gag & Seminoma & HERV-K & $(80,81)$ \\
GSDML & Uterine cervix cancer & HERV-H & $(82)$ \\
PTEN & Neoplasia & MMTV-LTR & $(10,42,83)$ \\
PTN & Choriocarcinoma and prostate cancer & HERV-E & $(84)$ \\
MID-1 & Embryonic kidney cell lines & HERV-E & $(38)$ \\
DNAJC15 & Several cancer cells & LTR33 and LTR7 & $(85)$ \\
PLA2L & Teratocarcinoma cells & HERV-H & $(39)$ \\
NAIP & Prostate & HERV-P & $(9)$ \\
CSF1R & Hodgkin's lymphoma & LTR & $(57)$ \\
PLA2G4A & Urothelial carcinoma & HERV-E & $(86)$ \\
CALB (Calbindin) & Prostate cancer cell line & HERV-H & \\
\hline
\end{tabular}

HERV, human endogenous retrovirus; LTRs, long terminal repeats.

enhancer activity (21). LTR may lead to polyadenylation of spliced chromosomal transcripts in human genes (HHLA2 and HHLA3) as it contains a poly(A) sequence (17). LTR can provide alternative and aberrant sites for transcript splicing and encode an additional carboxy-terminal sequence in the human leptin receptor (22). Similarly, LTR acts as one of at least two alternative promoters for the human $\beta 1,3$-galactosyltransferase 5 gene and is the dominant promoter in the colon (15). These functions of LTR are essential for LTR to regulate adjacent gene expression.

The most abundant transcriptional activation of HERV LTR may be observed in the placenta and embryonic tissues. Early studies of LTR transcript in placenta emphasized the expression of primary placenta of LTR of different HERV families [ERV3 (23); HERV-F (24); HERV-W $(25,26)]$. For example, 5'LTR regulates the expression of its downstream viral env gene in placenta trophoblast (26), while the ERV-9 regulatory region acts as a promoter to form a novel transcript of p63 in order to replenish cutaneous epithelial stem cells and maintain the fidelity of the female germ line (27). Further examples include the placenta-specific and tissue-specific expression of adjacent genes driven by LTR (28). These findings suggest that HERV LTR expression in human placenta is frequently active in human physical biology.

\section{Activation of HERV LTR in tumorigenesis}

Activation of HERV LTR in non-tumor disease. LTR activation is involved in diseases, such as rheumatoid arthritis $(29,30)$, type I diabetes (30), and schizophrenia (31). For example, the presence of LTR of the HERV-K family is associated with certain DQB alleles. Linkage disequilibrium with DQB1 alleles can contribute to susceptibility to rheumatoid arthritis (29). Expression of the GABBR1 gene is downregulated by HERV-W LTR in schizophrenia (31). Two members of the HERV-I family induce AZFa gene microdeletions in azoospermia patients (32). These abovementioned reports demonstrate that HERV LTR may be active in many diseases.

Activation of HERV LTR in tumor. Previous studies (33-37) have described the detection of RNA transcripts from various HERV LTRs in various types of human tumors and cell lines. Elevated HERV-K 5'LTR mRNA is significantly associated with tested prostate cancer tissues (33). It is also reported that the transcripts of the HERV-H LTR-derived promoter are widely distributed in various human tissues and cancer cells (11). Based on the function of LTRs on their adjacent genes in physiology described above, the function of the adjacent gene was investigated in order to elaborate the role of the LTR in tumorigenesis. The cancer-related LTR adjacent genes include viral and cellular genes. Considerable cancerassociated genes regulated by HERV LTR in tumorigenesis are shown in Table II. For example, the transcriptional start sites of HERV-K Rec and Np9 oncoproteins are regulated by 5'LTR (Table II). HERV-K Np9 is preferentially expressed in various tumor tissues (34) and the expression of Np9 significantly promoted the growth of leukemia cells in vitro and in vivo (35). HERV-K Rec expression was involved in the process of melanoma and germ cell tumor $(36,37)$. The GSDML gene with the alternative promoters from HERV-H LTR was able to promote 
cell proliferation and was correlated with carcinogenesis and the progression of uterine cervix cancer (Table II). DNAJC15 gene also possesses an alternative transcript provided by LTR33 and LTR7 elements. The LTR-related transcripts are only revealed in some cancer cells (HCT106, MCF-3, TE-1, HeLa and CCHM) compared to human tissues (38). Neuronal apoptosis inhibitory protein gene (NAIP), which contains LTR of endogenous retroviral elements as tissue-specific promoter, inhibits apoptosis in the neuron (39). Thus, HERV LTR is capable of regulating the expression of tumor-related genes in different tumor tissues or cells.

\section{Effect of possible mechanisms of HERV LTR in cancer}

The role of HERV LTR in tumorigenesis as well as the possible mechanisms of how HERV LTR contributes to the development of human cancer was investigated. An outline of potential mechanisms of HERV LTR in human tumorigenesis is provided in Fig. 2. The four aspects of genetic instability, methylation, transactivation and RNA interference by antisense transcripts are discussed below.

Genetic instability. Genetic instability is one of the key features associated with cancer causation and progression $(40,41)$. Instability exists at two distinct levels. In a small subset of tumors, instability is observed at the nucleotide level and results in base substitutions, deletions or insertions of a few nucleotides, such as polymorphisms. In the majority of other cancers, instability is observed at the chromosome level, resulting in losses and gains of whole chromosomes or large portions, for example insertion mutation and recombination. As a mobile element HERV LTR possesses the feature of genetic instability. Therefore, HERV LTR affects human genome instability involvement in tumorigenesis via three principal mechanisms: insertion, recombination and polymorphism (Fig. 2).

Retroviruses mediate malignant transformation via insertional mutagenesis or expression of viral genes. Similarly,HERV is also involved in human tumorigenesis via the mechanism of insertion (42). HERV LTR amplifies in the human genome by generating new integration events. The amplification of HERV integration in the human genome consists of three processes: replication, excision and transduction (Fig. 2). Insertions are therefore considered beneficial, negative or neutral. HERV LTR elements insert into genomic regions and contain crucial biological functions, resulting in insertional mutagenesis during evolution. Abnormal expression of novel gene and adjacent gene are also caused by the insertion of LTR. Among the examples of human cancers caused by LTR-mediated insertion mutagenesis, the expression of PTN gene contributes to the highly aggressive growth of human choriocarcinoma (42). LTR-associated insertional mutagenesis can contribute to cancer via germ-line and somatic mutations, either of which can directly lead to the onset of malignant transformation.

Solitary LTR is formed by homologous recombination between two full-length HERV families. A variety of different recombination products are shown in Fig. 2 (43), including recombination between the two LTRs of a single provirus, homologous recombination between two HERV on the same chromosome, recombination between the $3^{\prime}$ and 5'LTRs of one HERV and gene conversion between the non-homologous genes. Homologous recombination between two proviruses results in substantial deletion and rearrangements of cellular DNA. Gene conversion leads to non-homologous gene exchange with no proviral loss. This occurs predominantly through contributions to recombination, which is frequently detected in cancer (44). LTR33 and LTR7 are formed by recombination and can regulate DNAJC15 gene expression in cancer cells (38). Therefore, LTR recombination may be crucial for LTR to be involved in cancer progression.

The existence of polymorphism provides one explanation of how a ubiquitous gene causes disease in only a proportion of individuals. The polymorphism is subdivided into two broad categories: sequence and insertional polymorphisms. Only a few sequence polymorphisms have been described, partly due to the difficulty in identifying them against the background of closely similar proviruses. Using specific PCR primers spanning 5'LTRs of K115 and K113, Jha et al (45) reported the presence of three single nucleotide polymorphism sites in the K113 5'LTR and four in the K115 5'LTR that together constituted four haplotypes for K113. At present, there is little convincing evidence that any sequence polymorphism of a HERV provides susceptibility to human disease. For insertion polymorphisms, although most HERV families became integrated into the human genome millions of years ago, a new class of insertionally polymorphic HERV-K family members has recently been described $(46,47)$. HERV-K113 and HER-K115 insertion has been reported to be involved in various types of cancer (48-51). New insertion polymorphism of HERVs could serve as novel genetic risk factors and thus provide new insight for research into HERV LTR and cancer.

LTR affects the expression of adjacent genes by hypomethylation. Genetic instability of LTR leads to the its wide distribution in the human genome, while the activation of LTR can be controlled by DNA methylation. For example, Gimenez et al (52) have recently reported that hypomethylation of the promoter domain of the HERV U3 element appears to be a prerequisite for the increased expression in tumor tissues compared to normal tissues. Colon cancer cells are treated with DNA methylation and histone deacetylase inhibitors, and RT-PCR results show that the expression patterns of HERV-H are significantly altered in several colon cancer cells. The finding suggests that the hypomethylation context affects the expression of HERV-H elements in colon cancer cells (53). Increased HERV-K expression in melanomas may be due to increased promoter activity and demethylation of the 5'LTR (54). Thus, overexpression of the HERV sequence in cell lines is correlated with the demethylation of LTR.

LTR affects the expression of adjacent genes by RNA interference. HERV and LTR are usually oriented opposite to the transcription direction of corresponding host genes (55-57). Antisense transcripts affect the sense partner gene function by modifying the transcriptional and post-transcriptional regulation processes. Findings of a previous study (55) demonstrated that from 10 HERV-K LTRs which were localized in introns of unique human genes, nine exhibited opposite orientation to the transcription direction of the corresponding human genes. A hypothesis was propounded 
that LTR affects the gene expression by initiation of the antisense RNA synthesis (55). Subsequently, several reports supported the hypothesis (57-61). Antisense transcript of HERV-Ec1 affected the expression of cytosolic phospholipase A2 in urothelial carcinoma (57). A novel exon cassette is derived from the antisense transcript of the HERV-K element (58). Intronic RNAs arising from U3 of ERV-9 LTR are expressed as both sense and antisense transcripts, with the antisense transcript being expressed at higher levels compared to the sense expression in malignant cells (59). LTR from exogenous retrovirus HTLV can also generate antisense manuscripts such as the HTLV-1 basic leucine zipper factor (HBZ) (60). It has been reported that HBZ is consistently expressed and remains intact in all ATL cases and HTLV-1-infected individuals, where it promotes cell proliferation (61). Antisense transcripts are able to form double-stranded RNA and may recruit RNAi machinery. The abovementioned studies suggest that the abnormal expression of antisense transcripts of LTR retrotransposon may be a causative factor for tumorigenesis.

Transactivation of LTR. HERV LTR could also be reactivated by environmental factors including cytokines (62), radiation $(63,64)$ and proteins of exogenous retroviruses $(65,66)$. Radiation induced the epigenetic regulation of HERV-R 5'LTR and upregulated HERV-R env expression (63). HSV-1 activated the LTR activity of HERV-W to enhance their potential oligodendrotoxic and immunopathogenic effects (67). HSV-1 infection also induced the LTR-directed transcription of the HERV-K. HSV-1 immediate-early ICP0 protein was able to upregulate the activity of HERV-K LTR (66). Relative levels of transcripts encoding HERV-W elements and cellular genes are transactivated by viral infection in different cell lines by regulating the transcriptional activity (68). Several HERV LTRs could be activated by HTLV Tax and were involved in diseases (65). High-level expression of HERV-K has been demonstrated to be activated by the MITF-M gene in melanomas, breast cancers and teratocarcinomas (69). Of note, these factors can also target ERVs, for example, a HERV-K (HML-2) consensus element can be inhibited by APOBEC3F (70). Such LTR reactivation can enhance its impact on the surrounding genes in the pathological process of human cancer.

\section{Conclusion}

In summary, cumulative evidences indicate that LTR may be involved in the process of tumorigenesis at various levels. The potential role of LTR in human cancer appears much more complex (Fig. 2). LTR can be involved in tumorigenesis in four distinct ways, i.e., genetic instability, hypomethylation, transactivation and RNA interference. Insertional mutagenesis, recombination and polymorphism of LTR have been found to contribute to its genetic instability. Genetic instability can lead to diverse distribution and high copy numbers of LTR in the human genome. LTR can exhibit a sense or antisense orientation of their neighboring genes. By contrast, LTR can also be located in the 5'UTR, intron, exon and 3'UTR region of their neighboring genes. Genome-wide hypomethylation, transactivation of LTR and RNA interference by antisense orientation of LTR can regulate the expression of human cellular, viral and novel genes. Abnormal expression of cancer-associated genes may also contribute to tumorigenesis. Thus, identification of the distribution, structure and functional characteristics of LTR is extremely important to elucidate the mechanism involved in LTR regulation of cancer-associated gene abnormal expression in the process of tumorigenesis and development. Given the strong evidence for the abnormal expression of LTR adjacent genes in human tumors, it can be hypothesized that similar unknown genes or other tumor-associated genes may also affect various types of human cancer. Although a causative role of HERV LTR in human tumors has not been reported, HERV LTR is a potential contributory factor in various types of human cancer. Further investigations should include, for example, the novel insertion of HERV LTR in the human genome, RNA interference by antisense transcript of LTR, distribution of HERV LTR in different human chromosomes and the identification of LTR-related tumor genes.

\section{References}

1. Bannert N and Kurth R: Retroelements and the human genome: new perspectives on an old relation. Proc Natl Acad Sci USA 101 (Suppl 2): S14572-S14579, 2004.

2. de Parseval N and Heidmann T: Human endogenous retroviruses: from infectious elements to human genes. Cytogenet Genome Res 110: 318-332, 2005.

3. Leib-Mosch C, Haltmeier M, Werner T, et al: Genomic distribution and transcription of solitary HERV-K LTRs. Genomics 18: 261-269, 1993.

4. Tonjes RR, Lower R, Boller K, et al: HERV-K: the biologically most active human endogenous retrovirus family. J Acquir Immune Defic Syndr Hum Retrovirol 13 (Suppl 1): S261-S267, 1996.

5. Glazkova DV, Nadezhdin EV, Vinogradova TV, et al: Nucleotide sequences of long terminal repeats of the human endogenous retrovirus (LTR HERV-K) on the short arm of chromosome 7: identification, analysis and evaluation of transcriptional activity. Genetika 39: 702-708, 2003 (In Russian).

6. Kurdyukov SG, Lebedev YB, Artamonova II, et al: Full-sized HERV-K (HML-2) human endogenous retroviral LTR sequences on human chromosome 21: map locations and evolutionary history. Gene 273: 51-61, 2001.

7. Smit AF: Interspersed repeats and other mementos of transposable elements in mammalian genomes. Curr Opin Genet Dev 9: 657-663, 1999.

8. Belancio VP, Roy-Engel AM and Deininger PL: All y'all need to know 'bout retroelements in cancer. Semin Cancer Biol 20: 200-210, 2010.

9. Lamprecht B, Walter K, Kreher S, et al: Derepression of an endogenous long terminal repeat activates the CSF1R protooncogene in human lymphoma. Nat Med 16: 571-579, 2010.

10. Papadimitriou E, Mikelis C, Lampropoulou E, et al: Roles of pleiotrophin in tumor growth and angiogenesis. Eur Cytokine Netw 20: 180-190, 2009.

11. Sin HS, Huh JW, Kim DS, et al: Transcriptional control of the HERV-H LTR element of the GSDML gene in human tissues and cancer cells. Arch Virol 151: 1985-1994, 2006.

12. Feuchter A and Mager D: Functional heterogeneity of a large family of human LTR-like promoters and enhancers. Nucleic Acids Res 18: 1261-1270, 1990.

13. Medstrand P, Landry JR and Mager DL: Long terminal repeats are used as alternative promoters for the endothelin $\mathrm{B}$ receptor and apolipoprotein C-I genes in humans. J Biol Chem 276: 1896-1903, 2001

14. Dunn CA and Mager DL: Transcription of the human and rodent SPAM1/PH-20 genes initiates within an ancient endogenous retrovirus. BMC Genomics 6: 47, 2005.

15. Dunn CA, Medstrand P and Mager DL: An endogenous retroviral long terminal repeat is the dominant promoter for human beta1,3-galactosyltransferase 5 in the colon. Proc Natl Acad Sci USA 100: 12841-12846, 2003. 
16. Dunn CA, van de Lagemaat LN, Baillie GJ and Mager DL: Endogenous retrovirus long terminal repeats as ready-to-use mobile promoters: the case of primate beta3GAL-T5. Gene 364: 2-12, 2005

17. Mager DL, Hunter DG, Schertzer M and Freeman JD: Endogenous retroviruses provide the primary polyadenylation signal for two new human genes (HHLA2 and HHLA3). Genomics 59: 255-263, 1999.

18. Baust C, Seifarth W, Germaier H, Hehlmann R and LeibMosch C: HERV-K-T47D-Related long terminal repeats mediate polyadenylation of cellular transcripts. Genomics 66: 98-103, 2000.

19. Samuelson LC, Wiebauer K, Snow CM and Meisler MH: Retroviral and pseudogene insertion sites reveal the lineage of human salivary and pancreatic amylase genes from a single gene during primate evolution. Mol Cell Biol 10: 2513-2520, 1990.

20. Domansky AN, Kopantzev EP, Snezhkov EV, Lebedev YB, Leib-Mosch C and Sverdlov ED: Solitary HERV-K LTRs possess bi-directional promoter activity and contain a negative regulatory element in the U5 region. FEBS Lett 472: 191-195, 2000.

21. Ruda VM, Akopov SB, Trubetskoy DO, et al: Tissue specificity of enhancer and promoter activities of a HERV-K(HML-2) LTR. Virus Res 104: 11-16, 2004

22. Kapitonov VV and Jurka J: The long terminal repeat of an endogenous retrovirus induces alternative splicing and encodes an additional carboxy-terminal sequence in the human leptin receptor. J Mol Evol 48: 248-251, 1999.

23. Kato N, Pfeifer-Ohlsson S, Kato M, Larsson E, Rydnert J, Ohlsson R and Cohen M: Tissue-specific expression of human provirus ERV3 mRNA in human placenta: two of the three ERV3 mRNAs contain human cellular sequences. J Virol 61: 2182-2191, 1987.

24. Kjellman C, Sjogren HO, Salford LG and Widegren B: HERV-F (XA34) is a full-length human endogenous retrovirus expressed in placental and fetal tissues. Gene 239: 99-107, 1999.

25. Mallet F, Bouton O, Prudhomme S, et al: The endogenous retroviral locus ERVWE1 is a bona fide gene involved in hominoid placental physiology. Proc Natl Acad Sci USA 101: 1731-1736, 2004

26. Prudhomme S, Oriol G and Mallet F: A retroviral promoter and a cellular enhancer define a bipartite element which controls env ERVWE1 placental expression. J Virol 78: 12157-12168, 2004.

27. Liu M and Eiden MV: Role of human endogenous retroviral long terminal repeats (LTRs) in maintaining the integrity of the human germ line. Viruses 3: 901-905, 2011.

28. Landry JR and Mager DL: Functional analysis of the endogenous retroviral promoter of the human endothelin B receptor gene. J Virol 77: 7459-7466, 2003.

29. Seidl C, Donner H, Petershofen E, Usadel KH, Seifried E, Kaltwasser JP and Badenhoop K: An endogenous retroviral long terminal repeat at the HLA-DQB1 gene locus confers susceptibility to rheumatoid arthritis. Hum Immunol 60: 63-68, 1999.

30. Pascual M, Martin J, Nieto A, Giphart MJ, van der Slik AR, de Vries RR and Zanelli E: Distribution of HERV-LTR elements in the 5'-flanking region of HLA-DQB1 and association with autoimmunity. Immunogenetics 53: 114-118, 2001.

31. Hegyi H: GABBR 1 has a HERV-W LTR in its regulatory region - a possible implication for schizophrenia. Biol Direct 8 : $5,2013$.

32. Kamp C, Hirschmann P, Voss H, Huellen K and Vogt PH: Two long homologous retroviral sequence blocks in proximal Yq11 cause AZFa microdeletions as a result of intrachromosomal recombination events. Hum Mol Genet 9: 2563-2572, 2000.

33. Goering W, Ribarska T and Schulz WA: Selective changes of retroelement expression in human prostate cancer. Carcinogenesis 32: 1484-1492, 2011.

34. Armbruester V, Sauter M, Krautkraemer E, et al: A novel gene from the human endogenous retrovirus $\mathrm{K}$ expressed in transformed cells. Clin Cancer Res 8: 1800-1807, 2002.

35. Chen T, Meng Z, Gan Y, et al: The viral oncogene Np9 acts as a critical molecular switch for co-activating beta-catenin, ERK, Akt and Notch1 and promoting the growth of human leukemia stem/progenitor cells. Leukemia: Jan 11, 2013 (Epub ahead of print). doi: 10.1038/leu.2013.8

36. Boese A, Sauter M, Galli U, et al: Human endogenous retrovirus protein $\mathrm{CORF}$ supports cell transformation and associates with the promyelocytic leukemia zinc finger protein. Oncogene 19: 4328-4336, 2000
37. Singh S, Kaye S, Francis N, Peston D, Gore M, McClure M and Bunker C: Human endogenous retrovirus K (HERV-K) rec mRNA is expressed in primary melanoma but not in benign naevi or normal skin. Pigment Cell Melanoma Res 26: 426-428, 2013.

38. Sin HS, Huh JW, Kim DS, et al: Endogenous retrovirus-related sequences provide an alternative transcript of MCJ genes in human tissues and cancer cells. Genes Genet Syst 81: 333-339, 2006.

39. Romanish MT, Lock WM, van de Lagemaat LN, Dunn CA and Mager DL: Repeated recruitment of LTR retrotransposons as promoters by the anti-apoptotic locus NAIP during mammalian evolution. PLoS Genet 3: e10, 2007.

40. Lengauer C, Kinzler KW and Vogelstein B: Genetic instabilities in human cancers. Nature 396: 643-649, 1998.

41. Lin J, Gan CM, Zhang X, et al: A multidimensional analysis of genes mutated in breast and colorectal cancers. Genome Res 17: 1304-1318, 2007.

42. Schulte AM, Lai S, Kurtz A, Czubayko F, Riegel AT and Wellstein A: Human trophoblast and choriocarcinoma expression of the growth factor pleiotrophin attributable to germ-line insertion of an endogenous retrovirus. Proc Natl Acad Sci USA 93: 14759-14764, 1996.

43. Stoye JP: Endogenous retroviruses: still active after all these years? Curr Biol 11: R914-R916, 2001

44. Romanish MT, Cohen CJ and Mager DL: Potential mechanisms of endogenous retroviral-mediated genomic instability in human cancer. Semin Cancer Biol 20: 246-253, 2010.

45. Jha AR, Pillai SK, York VA, et al: Cross-sectional dating of novel haplotypes of HERV-K 113 and HERV-K 115 indicate these proviruses originated in Africa before Homo sapiens. Mol Biol Evol 26: 2617-2626, 2009

46. Turner G, Barbulescu M, Su M, Jensen-Seaman MI, Kidd KK and Lenz J: Insertional polymorphisms of full-length endogenous retroviruses in humans. Curr Biol 11: 1531-1535, 2001.

47. Dangel AW, Mendoza AR, Baker BJ, Daniel CM, Carroll MC, Wu LC and Yu CY: The dichotomous size variation of human complement $\mathrm{C} 4$ genes is mediated by a novel family of endogenous retroviruses, which also establishes species-specific genomic patterns among Old World primates. Immunogenetics 40: 425-436, 1994

48. Burmeister T, Ebert AD, Pritze W, Loddenkemper C, Schwartz S and Thiel E: Insertional polymorphisms of endogenous HERV-K113 and HERV-K115 retroviruses in breast cancer patients and age-matched controls. AIDS Res Hum Retroviruses 20: 1223-1229, 2004.

49. Wang-Johanning F, Radvanyi L, Rycaj K, et al: Human endogenous retrovirus $\mathrm{K}$ triggers an antigen-specific immune response in breast cancer patients. Cancer Res 68: 5869-5877, 2008.

50. Contreras-Galindo R, Kaplan MH, Leissner P, et al: Human endogenous retrovirus K (HML-2) elements in the plasma of people with lymphoma and breast cancer. J Virol 82: 9329-9336, 2008.

51. Golan M, Hizi A, Resau JH, Yaal-Hahoshen N, Reichman H, Keydar I and Tsarfaty I: Human endogenous retrovirus (HERV-K) reverse transcriptase as a breast cancer prognostic marker. Neoplasia 10: 521-533, 2008.

52. Gimenez J, Montgiraud C, Pichon JP, et al: Custom human endogenous retroviruses dedicated microarray identifies self-induced HERV-W family elements reactivated in testicular cancer upon methylation control. Nucleic Acids Res 38: 2229-2246, 2010.

53. Liang Q, Xu Z, Xu R, Wu L and Zheng S: Expression patterns of non-coding spliced transcripts from human endogenous retrovirus HERV-H elements in colon cancer. PLoS One 7: e29950, 2012.

54. Stengel S, Fiebig U, Kurth R and Denner J: Regulation of human endogenous retrovirus-K expression in melanomas by $\mathrm{CpG}$ methylation. Genes Chromosomes Cancer 49: 401-411, 2010.

55. Buzdin AA, Lebedev IuB and Sverdlov ED: Human genomespecific HERV-K intron LTR genes have a random orientation relative to the direction of transcription, and, possibly, participated in antisense gene expression regulation. Bioorg Khim 29: 103-106, 2003 (In Russian).

56. Li F, Nellaker C, Yolken RH and Karlsson H: A systematic evaluation of expression of HERV-W elements; influence of genomic context, viral structure and orientation. BMC Genomics 12: 22 , 2011.

57. Gosenca D, Gabriel U, Steidler A, et al: HERV-E-mediated modulation of PLA2G4A transcription in urothelial carcinoma. PLoS One 7: e49341, 2012. 
58. Kim DS and Hahn Y: Human-specific antisense transcripts induced by the insertion of transposable element. Int J Mol Med 26: 151-157, 2010

59. Xu L, Elkahloun AG, Candotti F, et al: A novel function of RNAs arising from the long terminal repeat of human endogenous retrovirus 9 in cell cycle arrest. J Virol 87: 25-36, 2012.

60. Gaudray G, Gachon F, Basbous J, Biard-Piechaczyk M, Devaux C and Mesnard JM: The complementary strand of the human T-cell leukemia virus type 1 RNA genome encodes a bZIP transcription factor that down-regulates viral transcription. J Virol 76 : 12813-12822, 2002.

61. Arnold J, Zimmerman B, Li M, Lairmore MD and Green PL: Human T-cell leukemia virus type-1 antisense-encoded gene, Hbz, promotes T-lymphocyte proliferation. Blood 112: 3788-3797, 2008.

62. Katsumata K, Ikeda H, Sato M, et al: Cytokine regulation of env gene expression of human endogenous retrovirus- $R$ in human vascular endothelial cells. Clin Immunol 93: 75-80, 1999.

63. Lee JR, Ahn K, Kim YJ, Jung YD and Kim HS: Radiationinduced human endogenous retrovirus (HERV)-R env gene expression by epigenetic control. Radiat Res 178: 379-384, 2012.

64. Reiche J, Pauli G and Ellerbrok H: Differential expression of human endogenous retrovirus $\mathrm{K}$ transcripts in primary human melanocytes and melanoma cell lines after UV irradiation. Melanoma Res 20: 435-440, 2010.

65. Toufaily C, Landry S, Leib-Mosch C, Rassart E and Barbeau B: Activation of LTRs from different human endogenous retrovirus (HERV) families by the HTLV-1 tax protein and T-cell activators. Viruses 3: 2146-2159, 2011

66. Kwun HJ, Han HJ, Lee WJ, Kim HS and Jang KL: Transactivation of the human endogenous retrovirus $\mathrm{K}$ long terminal repeat by herpes simplex virus type 1 immediate early protein 0 . Virus Res 86: 93-100, 2002.

67. Ruprecht K, Obojes K, Wengel V, et al: Regulation of human endogenous retrovirus $\mathrm{W}$ protein expression by herpes simplex virus type 1: implications for multiple sclerosis. J Neurovirol 12: 65-71, 2006.

68. Nellaker C, Yao Y, Jones-Brando L, Mallet F, Yolken RH and Karlsson H: Transactivation of elements in the human endogenous retrovirus W family by viral infection. Retrovirology 3 : 44, 2006.

69. Katoh I, Mirova A, Kurata S, et al: Activation of the long terminal repeat of human endogenous retrovirus $\mathrm{K}$ by melanoma-specific transcription factor MITF-M. Neoplasia 13: 1081-1092, 2011.

70. Lee YN and Bieniasz PD: Reconstitution of an infectious human endogenous retrovirus. PLoS Pathog 3: e10, 2007.

71. Lee JW and Kim HS: Endogenous retrovirus HERV-I LTR family in primates: sequences, phylogeny, and evolution. Arch Virol 151: 1651-1658, 2006.
72. Yi JM and Kim HS: Evolutionary implication of human endogenous retrovirus HERV-H family. J Hum Genet 49: 215-219, 2004.

73. Mager DL and Henthorn PS: Identification of a retrovirus-like repetitive element in human DNA. Proc Natl Acad Sci USA 81: 7510-7514, 1984

74. Blond JL, Beseme F, Duret L, et al: Molecular characterization and placental expression of HERV-W, a new human endogenous retrovirus family. J Virol 73: 1175-1185, 1999.

75. Gifford R and Tristem M: The evolution, distribution and diversity of endogenous retroviruses. Virus Genes 26: 291-315, 2003.

76. Strazzullo M, Parisi T, Di Cristofano A, Rocchi $M$ and La Mantia G: Characterization and genomic mapping of chimeric ERV9 endogenous retroviruses-host gene transcripts. Gene 206: 77-83, 1998.

77. Tonjes RR, Czauderna F and Kurth R: Genome-wide screening, cloning, chromosomal assignment, and expression of full-length human endogenous retrovirus type K. J Virol 73: 9187-9195, 1999.

78. Wang-Johanning F, Frost AR, Jian B, Epp L, Lu DW and Johanning GL: Quantitation of HERV-K env gene expression and splicing in human breast cancer. Oncogene 22: 1528-1535, 2003.

79. Herbst H, Sauter M and Mueller-Lantzsch N: Expression of human endogenous retrovirus $\mathrm{K}$ elements in germ cell and trophoblastic tumors. Am J Pathol 149: 1727-1735, 1996.

80. Huh JW, Kim DS, Kang DW, et al: Transcriptional regulation of GSDML gene by antisense-oriented HERV-H LTR element. Arch Virol 153: 1201-1205, 2008.

81. Sun Q, Yang J, Xing G, Sun Q, Zhang L and He F: Expression of GSDML associates with tumor progression in uterine cervix cancer. Transl Oncol 1: 73-83, 2008.

82. Backman SA, Ghazarian D, So K, et al: Early onset of neoplasia in the prostate and skin of mice with tissue-specific deletion of Pten. Proc Natl Acad Sci USA 101: 1725-1730, 2004.

83. Tsirmoula S, Dimas K, Hatziapostolou M, Lamprou M, Ravazoula P and Papadimitriou E: Implications of pleiotrophin in human PC3 prostate cancer cell growth in vivo. Cancer Sci 103: 1826-1832, 2012

84. Landry JR, Rouhi A, Medstrand P and Mager DL: The Opitz syndrome gene Mid1 is transcribed from a human endogenous retroviral promoter. Mol Biol Evol 19: 1934-1942, 2002.

85. Kowalski PE, Freeman JD and Mager DL: Intergenic splicing between a HERV-H endogenous retrovirus and two adjacent human genes. Genomics 57: 371-379, 1999.

86. Gebefügi E, Brunmeir R, Weierich C, Wolff H, Brack-Werner R and Leib M: Activation of a HERV-H LTR induces expression of an aberrant calbindin protein in human prostate carcinoma cells. Retrovirology 6 (Suppl 2): P48, 2009. 